American Journal of Applied Sciences 3 (5): 1824-1830, 2006

ISSN 1546-9239

(c) 2006 Science Publications

\title{
Mixed Mode EMI Noise Level Measurement in SMPS
}

\author{
${ }^{1}$ R.Dhanasekaran, ${ }^{1}$ M.Rajaram and ${ }^{2}$ S.N.Sivanandam \\ ${ }^{1}$ Department of Electrical Engineering, Govt. College of Technology, Coimbatore-641013, TN, India \\ ${ }^{2}$ Department of CSE, P.S.G. College of Technology, Coimbatore, TN, India
}

\begin{abstract}
Fast switching in SMPS generate large amount of Electromagnetic Interference (EMI).The proposed method to determine Common Mode (CM) and Differential Mode (DM) noise of a low power Switched Mode Power Supply (SMPS) using two-probe approach i.e. using one current probe as an injecting probe and other current probe as receiving probe Common Mode (CM) noise is measured between power line and ground. Differential Mode (DM) noise is measured between each power lines. EMI radiated emissions occur in the range of $150 \mathrm{KHz}-30 \mathrm{MHz}$. EMI filter is usually needed in the input of the Switch Mode Power Supply (SMPS) to attenuate the noise. The proposed approach allows measurement of noise level in Switched Mode Power Supply without interleaving its normal operation. With proper set up calibration, the proposed approach can be used to measure the noise level with reasonable accuracy. The noise level is measure from the frequency of $1 \mathrm{MHz}-3 \mathrm{MHz}$ using signal generator the signal is induced in the injected probe and using receiving probe noise level is measured in $\mathrm{db}$ using spectrum analyser.
\end{abstract}

Key words: Common mode (CM), differential mode (DM), LISN, EUT, spectrum analyser

\section{INTRODUCTION}

In the field of power electronics, there is a trend for pushing up switching frequencies of Switched Mode Power Supplies to reduce volume and weight. This trend inevitably contributes to an increasing level of Electro Magnetic Interference (EMI) emissions. It leads to a general Electromagnetic Compatibility (EMC) degradation for electronic devices. Electromagnetic Interference (EMI) problems in Switching Power Supplies have been traditionally treated with cut-andtry approaches. In recent years, advancement has been made to better understand the problems and minimize the cut-and-try portion of the design process.

Conventionally, the total conducted EMI noise is caused by two mechanisms, the Differential Mode (DM) and Common Mode (CM) Noise. Generally speaking, the Differential Mode (DM) noise is related to Switching Current and the Common Mode (CM) noise is related to Capacitive Coupling of switching voltage into Line Impedance Stabilizing Network (LISN), which is used in standard conducted EMI measurement. Figure 1 shows the typical setup for conducted EMI measurement. The LISN contains inductors, capacitors and 50 ?resistors.

For $50 \mathrm{~Hz}$ line frequency, the inductors are basically shorted; the capacitors are open and the power passes through to supply the Equipment Under Test (EUT). For EMI noise frequency, the inductors are essentially open, the capacitors are shorted and the noise sees $50 \Omega$ resistors. The noise voltage measured across the $50 \Omega$ input impedance of a Spectrum

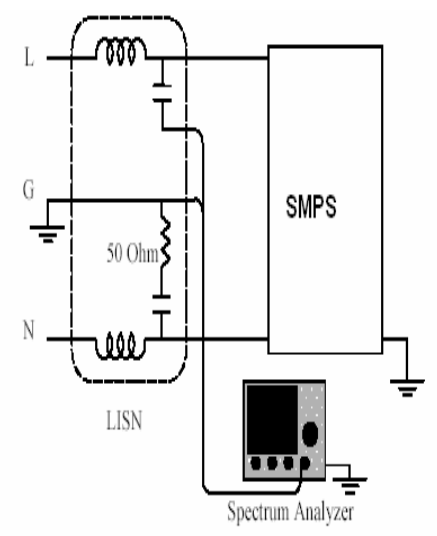

Fig.1: Basic set up of $\mathrm{CM}$ and DM noise

Analyzer is defined as the conducted EMI emission. There are two types of measuring noise, one is Resonance Method and another one is Insertion loss method.

Resonance Method has been employed to estimate the noise source impedance of a SMPS by terminating the power input of the SMPS with a reactive component that is of opposite type to the noise source reactance. When the frequency of measurement is high the parasitic effect of non-ideal reactive components become significant and the circuit topology based on which the resonance method is no longer valid. Incase of Differential Mode (DM) noise source impedance measurement, attempt of achieve parallel LC resonant are very difficult because of the low impedance nature of the noise source. The main disadvantages of the Resonance Method are the process of choosing the right

Corresponding Author: R.Dhanasekaran Teaching Research Associate Department of Electrical Engg. Govt.College of Technology Coimbatore - 641 013.TN,INDIA 
values of reactive components and to tune for resonance can be very tedious.

Insertion Loss Method is also been introduced to measure the noise source impedance of a SMPS.Here the main disadvantages the impedance of the inserted component is much larger or smaller than the noise source impedance.

Two Probe Approach is to overcome the problems faced be the previous methods, a new approach called two current probe approach is developed to measure, the noise level in $\mathrm{db}$ and its attenuation of SMPS.

Determination of Common Mode (CM) and Differential Mode (DM) Noise In SMPS, EMI filter design for both Common Mode (CM) and Differential Mode (DM) noise, Filter termination impedance ${ }^{[1]}$. Design for Electromagnetic Compatibility (EMC) has become a requirement. EMC's is the absence of EMI. Electromagnetic Compatibility (EMC) is a system level consideration. This topic attempts to describe the more significant causes of EMI in power supplies and offer design techniques to minimize their impact ${ }^{[2]}$. Discuss about the sources and paths of Common Mode (CM) and Differential Mode (DM) noise, which is due the rapid frequency changes ${ }^{[3]}$. Discuss about the equivalent circuit of noise source for an Off-Line Converter and design an EMI Filter ${ }^{[4,5]}$. States the measurement of noise source impedance of Off-Line Converter $^{[5]}$. Measuring of noise source impedance of Common Mode (CM) and Differential Mode (DM) Noise Using Two Probe Approach ${ }^{[6]}$.

Referred the Block of SMPS and the Filter Design and Common Mode (CM) and Differential Mode (DM) noise ${ }^{[7]}$. Referred for the filter design and reduction of Common Mode (CM) and Differential Mode (DM) noise in SMPS ${ }^{[8]}$. Collected the details about Common Mode (CM) and Differential Mode (DM) NOISE, LISN, Spectrum Analyzer and EMI Filter ${ }^{[9,10]}$.

EMI in SMPS: Switched Mode Power Supplies (SMPS) are usually a part of a complex electronic system. The system operates with electric signals with much lower amplitude and energy compared to those on an SMPS. It means that usually the SMPS is the strongest electrical noise generator in the whole system.

Especially the power switches with their high $d v / d t$ and $d i / d t$ switching slopes are the sources of EMI. The source of Differential Mode (DM) Interferences is the current switched by a MOSFET or a diode. High rates of $d v / d t$ and parasitic capacitors to the ground are the reasons for Common Mode Interferences.

Conventional conducted EMI in SMPS: A typical Offline Switching Power Supply with conducted EMI measurement setup. A Line Impedance Stabilizing Network (LISN) is used for the measurement of conducted EMI emissions. LISN is used as a standard for repeatable EMI measurement. The standard components inside the LISN are such that for $50 \mathrm{~Hz} \mathrm{AC}$

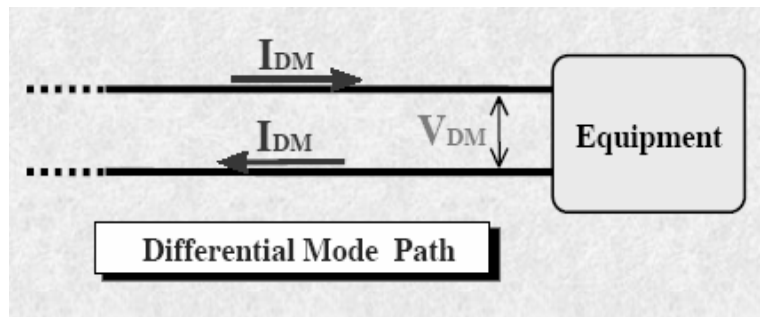

Fig. 2: Differential mode current path

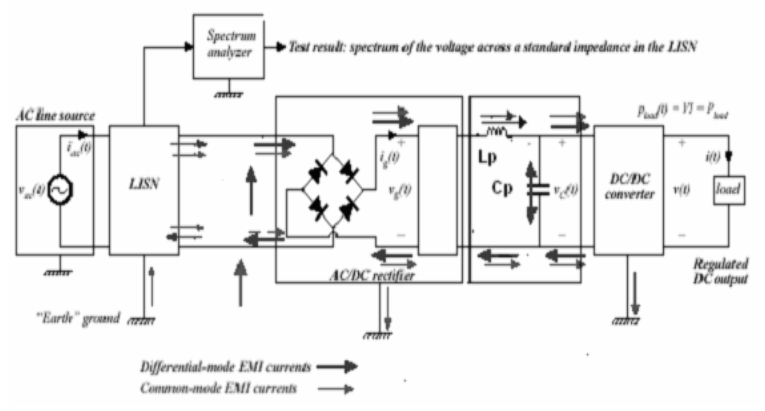

Fig. 3: Differential and common mode EMI

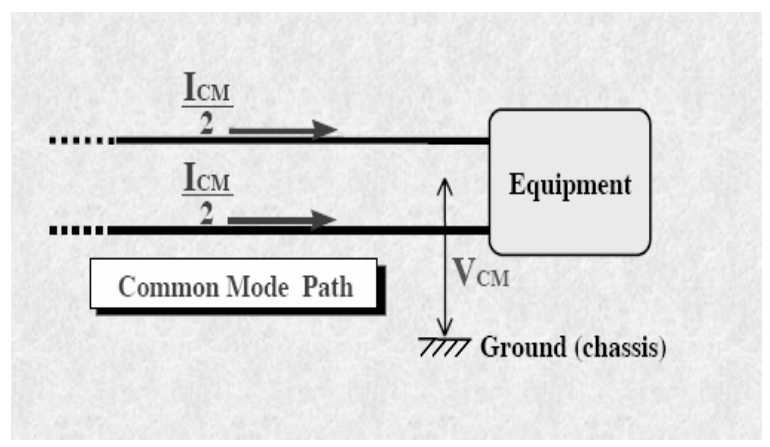

Fig. 4: Common mode current path

power, the inductance $(10 \mathrm{mH})$ presents very small impedance and capacitor $(0.1 \square \mathrm{F})$ is essentially open circuit. Therefore, the $50 \mathrm{~Hz}$ power flows unperturbed. For high frequency noise, however, the inductance looks like open circuit and the capacitor is basically short circuit.

Because of the switching nature of the circuit, high frequency noise current could be coupled into the $50 \Omega$ resistors. The voltage across the $50 \Omega$ resistors is counted as the conducted EMI emission. Both the line side $(\mathrm{Vx})$ and the neutral side (Vy) EMI must pass specs. The noise is classified into two modes: Common-Mode (CM), which is average of $\mathrm{Vx}$ and $\mathrm{Vy}$ $((V x+V y) / 2)$ and Differential-Mode (DM) which is the difference between $\mathrm{Vx}$ and $\mathrm{Vy}(\mathrm{Vx}-\mathrm{Vy})$.

EMI types in SMPS: EMI is transmitted in two forms: Radiated noise and Conducted noise Radiated Noise occurs in the range of $30 \mathrm{MHz}-1 \mathrm{GHz}$.Radiated Noise requires the measurement of magnetic or electric fields in free space, causing the testing to become much more complex. 
The conducted EMI is measured with Line Impedance Stabilization Network (LISN), in the frequency range of $150 \mathrm{kHz}$ to $30 \mathrm{MHz}$.LISN is used to provide the $50 \Omega$ standard impedance for the measurably repeatability. Conducted Noise is higher than Radiated Noise. Conducted Noise consist of two categories commonly known as Differential Mode Noise(DM) and Common Mode Noise (CM)

Differential mode noise (DM): It is measured between each power line and ground. Differential Mode (DM) is due to magnetic coupling. It is otherwise called as Normal-Mode or Transverse-Mode Noise. Current path of differential mode is shown in Fig. 2 Differential Mode (DM) noise attempts to dissipate its energy along any path from line to neutral. If the Normal-Mode noise has sufficient voltage (or energy), damage could first occur to the SMPS and then to the computer circuitry. The $\mathrm{p}-\mathrm{n}$ junction of the rectified diodes can breakdown due to excessive biasing. The capacitors may degrade if the noise is opposite in polarity or exceeds operating limits. Transformer insulation may breakdown if the noise peaks are excessively high. The transmission of the Differential Mode (DM) noise is through the input line to the utility system and through the dc-side network to the load on the power converter. Differential Mode (DM) noise is presents on both the input and output lines.

Conducted EMI noise coupling through the $50 \Omega$ resistors is shown in Fig. 3 Because of the switching nature of the thyristor current, part of the switch current flows through the bulk capacitor $\mathrm{C}_{\mathrm{p}}$ and part through the $50 \Omega$ resistors as indicated by the dash line. Since $C_{p}$ is not perfect due to the existence of parasitic inductance $L_{p}$, there is certain amount of noise current flowing through the $50 \Omega$ resistors. Notice that the current flows through the resistors in series. In general, the Differential Mode (DM) noise current is load dependent and is affected by $\mathrm{L}_{\mathrm{p}}$ and $\mathrm{C}_{\mathrm{p}}$.

Common mode noise (CM): Common Mode (CM) noise is measured between LINE and GROUND. Common Mode (CM) noise is due to stray capacitance.

Common Mode (CM) noise may be coupled through the high frequency transformer or along paths that have parasitic or stray capacitance. It consists of high frequency impulses, there is a high probability that the noise will see the high frequency transformer just as a coupling capacitor and pass through unobstructed. Stray Capacitor paths may exist within SMPS because they are smaller in physical size and more densely packaged as compared to other types of power supplies. Common Mode (CM) noise is present on both input and output lines. The current path of common mode is shown in Fig. 4. The transmission of Common Mode (CM) noise is entirely through "parasitic" or stray capacitors and stray electric and magnetic fields.
These stray capacitances exist between various system components and between component and ground.

The Common Mode (CM) noise is coupled through $\mathrm{C}_{\mathrm{p}}$, the parasitic capacitance between the drain of the thyristor, since the thyristor is operated as a switch, the drain voltage swings from low to high in half of the switching cycle and from high to low in the second half of the switching cycle. This voltage swing in turn causes the charging and discharging of the parasitic capacitance. The charging and discharging current will return through the ground path and show up on the LISN resistors as $\mathrm{CM}$ noise.

The Common Mode (CM) noise path is illustrated with thin line in Fig. 3. Notice that the noise current flows through the two $50 \Omega$ resistors in parallel. Figure 3 shows the current path when $C_{p}$ is charged. When $C_{p}$ is discharged, the current direction reverses but both currents flow in phase. Therefore, it is called Common Mode (CM) Noise. In general, Common Mode (CM) noise is voltage dependent and depends on parasitic capacitance. Besides the parasitic capacitance of thyristor, there are other parasitic capacitors (such as the parasitic capacitance between the transformer primary and secondary windings and the parasitic capacitance of the secondary rectifier diode), through which the CM noise current can couple.

\section{BASIC THEORY OF THE TWO CURRENT PROBE APPROACH}

The two current probe consist of one current probe as an injection probe and another current probe as a receiving probe, to measure Common Mode (CM) and Differential Mode (DM) noise level in db with EMI regulated frequency range of any SMPS.

Magnitude of noise measurement of SMPS using the two current probe approach is shown in Fig. 5.The components involves an injecting current probe, a receiving current probe, a signal generator and a spectrum analyzer.

The unknown impedance to be measured is represented by $Z_{x}$ at $b-b$. The two current probes and a coupling capacitors form a radio frequency (RF) coupling circuit for the measurement of magnitude of $\mathrm{Z}_{\mathrm{x}}$. The coupling capacitor $\mathrm{C}$ has an Equivalent Series Resistance (ESR) of $\mathrm{R}_{\mathrm{C}}$ and Equivalent Series Inductance $(\mathrm{ESL})$ of $\mathrm{L}_{\mathrm{C}}$. A continuous wave $(\mathrm{CW})$ signal $\mathrm{V}_{\mathrm{w}}$ is induced into the coupling circuit through the signal generator and the Injecting Current Probe. The Receiving Current Probe, the Spectrum Analyzer measures the magnitude of the resultant current $I_{w}$ in the wire of the coupling circuit. The measurement will be made at a series of frequency within the EMI regulated frequency at a series of frequency range of $150 \mathrm{kHz}$ to $30 \mathrm{MHz}$. At each frequency, by adjusting the signal generator to the right signal output level, 


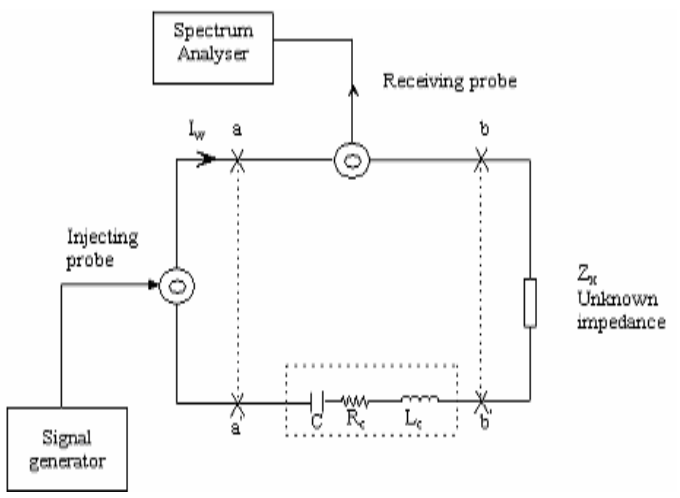

Fig. 5: Basic set up of two probes

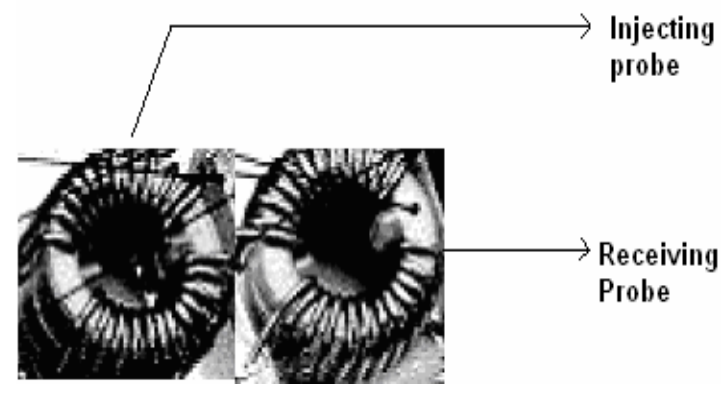

Fig. 6: Injecting probe and receiving probe

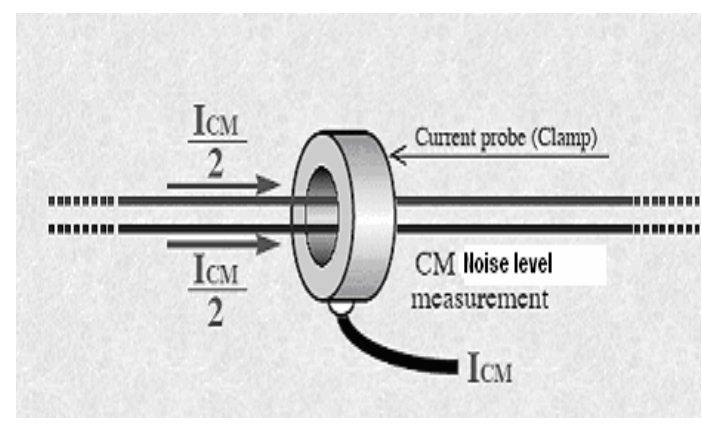

Fig. 7: Line and neutral inserted into the probe for $\mathrm{CM}$ noise measurement

the induced signal from the injecting current probe can be detected by the Spectrum Analyzer via receiving probe.

Conducted emission sensors reviewed in this section are the current probe, the line impedance stabilization network (LISN) sometimes called artificial mains and the voltage probe. The conducted injectors reviewed, cover special transformers and impedancematching devices to permit injecting a specified voltage across or current into lines for susceptibility testing.

In general, either the current probe or the LISN can be used to measure conducted power line emissions depending upon which is required. Sometimes both are used together. The current and voltage probe, on other hand, are both useful for measuring conducted signal and control-line emissions appearing on equipment

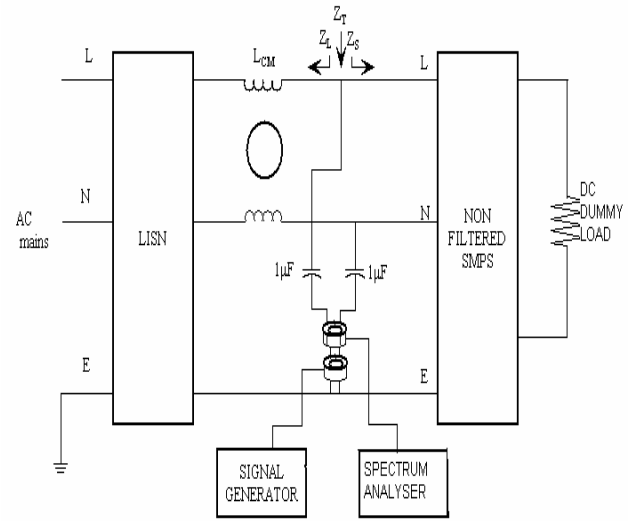

Fig. 8: CM noise measurement setup

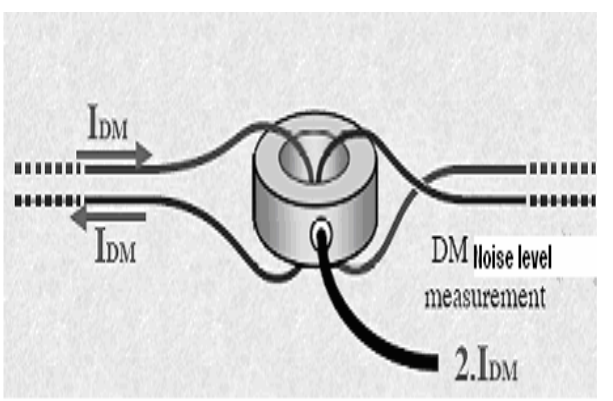

Fig. 9: Line is inserted into the probe for DM noise measurement

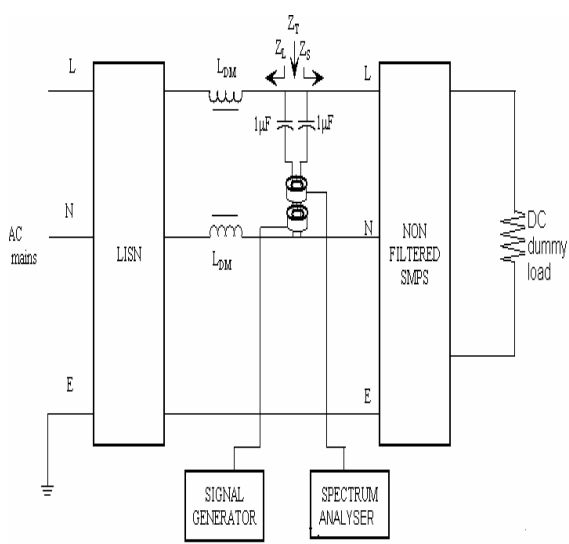

Fig. 10: DM noise measurement set up

interconnecting wires and cables. The equivalent EMI voltage is simply the current probe measurement.

Current probes work on the principle of sampling the magnetic field around a wire (the primary) by secondary turns on a toroid transformer. The induced voltage in the output of the current probe is proportional to the permeability of the toroid, its cross sectional area, number of secondary turns, the current flowing in the wire and frequency. The transfer impedance increasing with the frequency at a rate of 20 $\mathrm{db}$ per decade. thus to make a sensitive current probe, especially at lower frequency, the permeability,area, and number of turns on the toroid should be made as large 
as practical with a relatively small toroid radius. The Fig. 6 shows the injecting probe and receiving probe.

Measurement of CM noise: The Common Mode (CM) setup is shown in Fig. 8. The test setup is similar to Differential Mode (DM) setup. The current probe is configured to measure the Common Mode Emission. LISN is used to measure the Common Mode Noise. The probe is connected between LISN and SMPS. The noise is measured in line to ground.The two $1 \square \mathrm{F}$ capacitors together with the injecting and receiving current probes form the RF coupling circuit. The advantage of fixing the coupling circuit is that once it is calibrated to obtain $\mathrm{Z}_{\mathrm{x}}$, it can be used in Common Mode (CM) to speed up the noise source impedance measurement process. Figure 7 line and neutral wire inserted into the probe for noise measurement

$\mathrm{Z}_{\text {in }}$ of the coupling circuit can be modeled as $1.12 \Omega$ resistor, a $240 \mathrm{nH}$ inductor and $2.2 \square \mathrm{F}$ capacitors in series. $\mathrm{KV}_{\text {sig }}$ and $\mathrm{Z}_{\text {in }}$ are found, the coupling circuit is ready to measure any unknown impedance. By observing the change in the measured magnitude of $\mathrm{Z}_{\text {in }}$ from $10 \mathrm{KHz}$ to $30 \mathrm{MHz}$. The actual measured impedance of Radio Frequency (RF) coupling circuit is $\mathrm{Z}_{\mathrm{T}}$, which is $\mathrm{Z}_{\mathrm{L}}$ and $\mathrm{Z}_{\mathrm{s}}$ in parallel, where $\mathrm{Z}_{\mathrm{s}}$ is the actual noise source to be measured and $Z_{s}$ is the RF isolation to be provided by a suitable choke. For common mode set up, a $16 \mathrm{mH}$ choke is inserted between the LISN and SMPS to provide RF isolation.

Measurement of DM noise: The Differential Mode (DM) setup is shown in Fig. 10. The test setup is similar to Common Mode setup. The current probe is configured to measure the Differential Mode (DM) Emission. LISN is used to measure the Differential Mode Noise. The probe is connected between LISN and SMPS. The noise is measured in line to line is shown in Fig. 9.

The two $1 \mu \mathrm{F}$ capacitors together with the injecting and receiving current probes form the RF coupling circuit. For Differential Mode (DM) set up, two 350 $\square \mathrm{H}$ $\mathrm{dm}$ chokes are added between the LISN and SMPS to provide DM RF isolation. The rated current for the selected DM noise chokes is $3 \mathrm{~A}$

The two current consist of one current probe as an injection probe and another current probe as a receiving probe, to measure Common Mode (CM) and Differential Mode (DM) noise source impedances EMI regulated frequency range of any SMPS. Magnitude of noise measurement of any unknown impedance using the two current probe approach is shown in Fig. 4. The components involves an injecting current probe, a receiving current probe, a signal generator and a spectrum analyzer. The unknown impedance to be measured is represented by $Z_{x}$ at b-b'. The two current probes and a coupling capacitors form a radio frequency $(\mathrm{RF})$ coupling circuit for the measurement of magnitude of $\mathrm{Z}_{\mathrm{x}}$. The coupling capacitor $\mathrm{C}$ has an

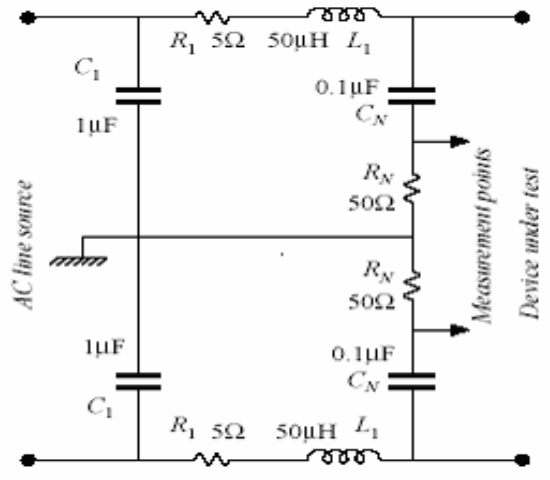

Fig 11: Line impedance stabilization network (LISN)

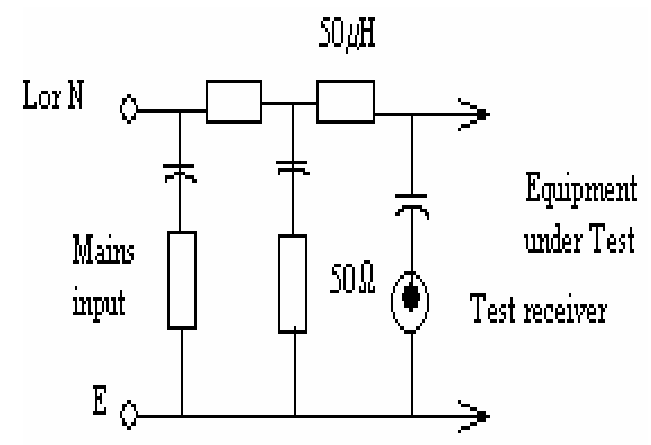

Fig. 12: LISN used for EUT

Equivalent Series Resistance (ESR) of $R_{C}$ and Equivalent Series Inductance (ESL) of $\mathrm{L}_{\mathrm{C}}$. A continuous wave $(\mathrm{CW})$ signal $\mathrm{V}_{\mathrm{w}}$ is induced into the coupling circuit through the signal generator and the Injecting Current Probe.

\section{Hardware Setup}

LISN: LISN is called Line Impedance Stabilization Network. A LISN's purpose is to provide stabilized impedance to conducted emissions, without interfering with the normal power flow required by the equipment under test (EUT). At the power line frequency the LISN provides a low impedance path from the power source to the load impedance and a high impedance path from the load to ground. The 50 impedance to ground is actually the input impedance of the spectrum or EMI meter used to measure the noise.

The line impedance stabilization network(LISN),sometimes called a line stabilization network(LSN), power-line impedance stabilization network(PLISN), or artificial mains is a coupling unit used to measure conducted emissions from a test sample power leads and not that coming from the power mains supply to these leads. such an installation is shown. in other words, the LISN is buffer network which permits connecting the power leads of the test item to the power mains by (1)passing only the DC or AC power to the test sample,(2) preventing the test sample's electromagnetic noise from getting back into the power bus,(3)blocking the power mains R-F from 
coupling into the test sample. The LISN provides for direct connection of the $50 \Omega$ input terminals of an EMI receiver to the $50 \Omega$ connector provided on the LISN.

The schematic diagram of the LISN shown in Fig. 11 , it is noted that the power leads from then power supply mains bus and those from the test specimen are $\mathrm{R}-\mathrm{F}$ isolation by the series inductance. it is transparent at 60 and $400 \mathrm{~Hz}$ to permit direct coupling of the power source to the test item-F noise originating on the power bus is shunted to ground through the coupling capacitor, Cp.conversely, any R-F noise on the test sample's power leads is coupled to the $50 \Omega$ connector jack through the capacitor.LISN is used for voltage measurements or it is used to isolate EMI and stabilize impedance while employing a current probe to measure current. The circuit is shown in Fig.12, one of these circuits is inserted in series with each of the live and neutral lines or, in the case of a three phase supply, in series with each of L1, L2 and L3.

\section{Specification of LISN used:}

Product code:

Rated Voltage:

at $50 \mathrm{~Hz}$

Rated Current:

Leakage Current:

High voltage test for 60 seconds:

CHOKE

For common mode choke:

For differential mode choke:

The choke is the inductance used in line and

neutral, connected after LISN

Spectrum analyzer is used for some conducted and radiated emission applications by the EMC and related communities. In contrast to EMI receivers, modern spectrum analysers are generally characterizer by unturned front ends, relatively high noise figures; builtin electro-optical (CRT) spectrum amplitude display unit with variable persistence.amplitute calibration of intercepted signals is achieved through narrow-band means. Most spectrum analysers offer little dynamic range to impulsive signals because of wide-open front ends. The principle advantage of the spectrum analyzer over EMI receivers is their flexibility and functional displays.

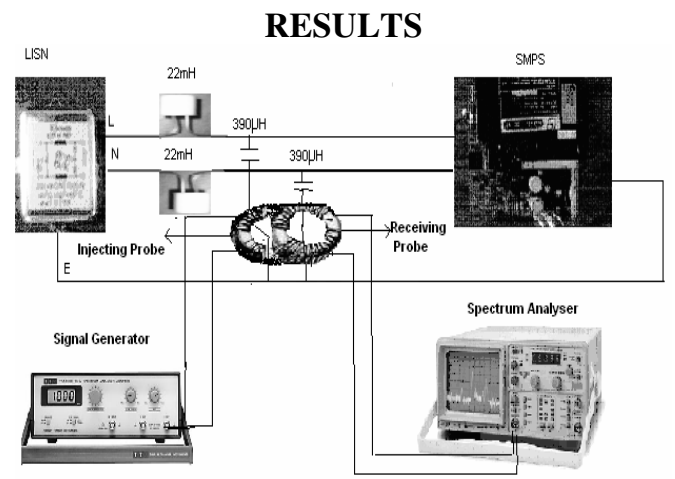

Fig. 13: Hardware set up of common mode noise measurement

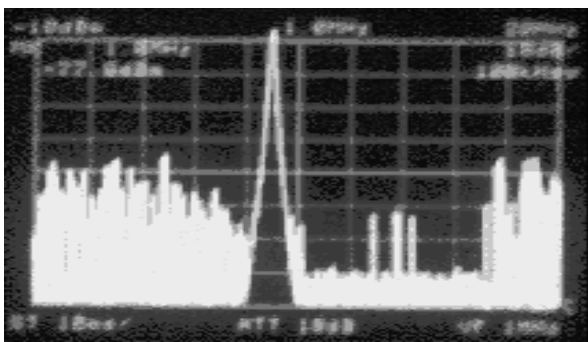

(a)

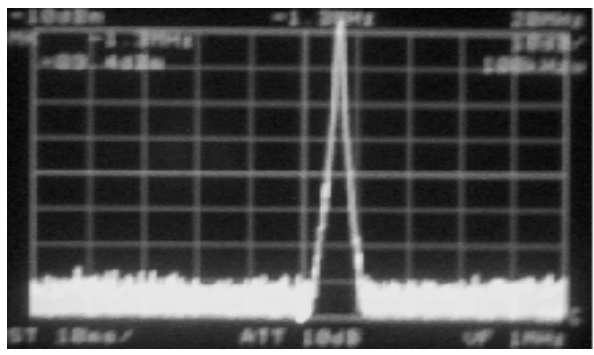

(b)

Fig. 14: Differential mode noise level measurement

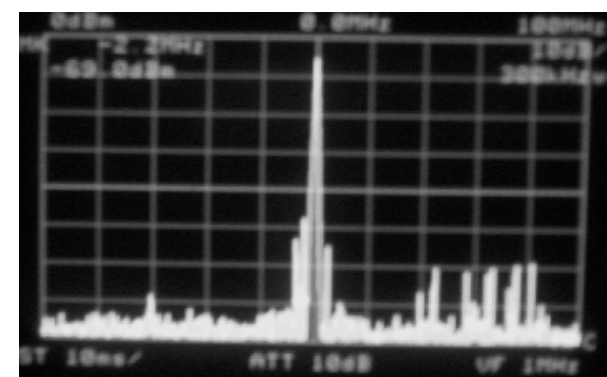

(a).3.2MHz, $33 \mathrm{~dB}$

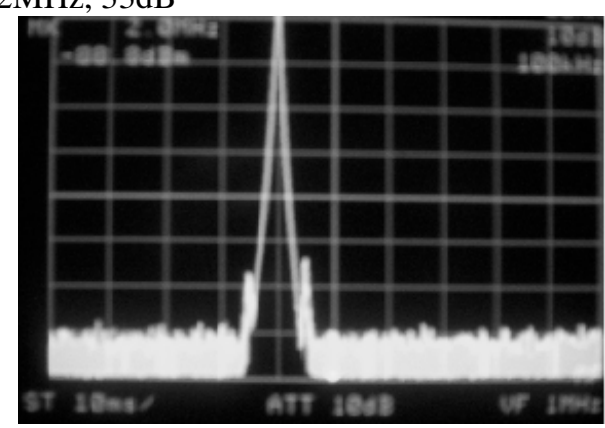

(a). $2 \mathrm{MHz}, 28 \mathrm{~dB}$

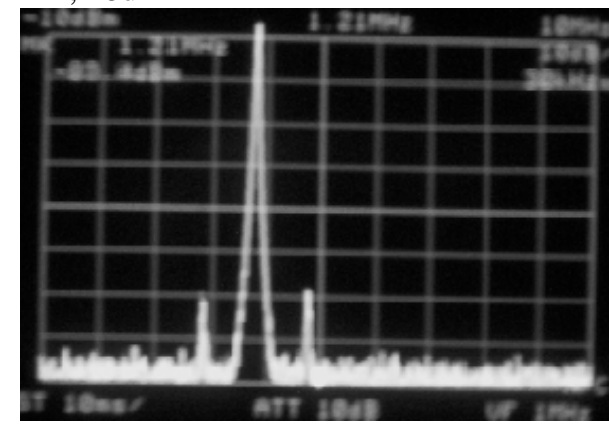

(b). 1.2MHz, $22 \mathrm{~dB}$

(a).Signal with Noise (b).Signal without noise

Fig. 15: Common mode noise level measurement 
Table1: Measured common mode and differential mode noise level in $\mathrm{dB}$

Common Mode Noise level in $\mathrm{dB}$

\begin{tabular}{lc}
\hline Frequency in $\mathrm{MHz}$ & Noise level in $\mathrm{dB}$ \\
\hline 1.0 & 20 \\
1.2 & 22 \\
1.5 & 23 \\
1.6 & 24 \\
2.0 & 28 \\
2.2 & 29 \\
2.7 & 30 \\
3.0 & 32 \\
3.2 & 33 \\
\hline
\end{tabular}

Differential Mode Noise level in $\mathrm{dB}$

\begin{tabular}{lc} 
Frequency in $\mathrm{MHz}$ & Noise level in $\mathrm{dB}$ \\
\hline 1.0 & 40 \\
1.2 & 30 \\
1.5 & 30 \\
1.6 & 29 \\
2.0 & 25 \\
2.2 & 23 \\
2.7 & 20 \\
3.0 & 19 \\
3.2 & 15 \\
\hline
\end{tabular}

\section{CONCLUSION}

The two probes set up allows measurement of the noise level of the Switch Mode Power Supply(SMPS) and its noise level has been is measured and displayed in Spectrum Analyzer under normal "power on" condition, for resistive loading conditions. For the frequency from $1 \mathrm{MHz}$ to $3.2 \mathrm{MHz}$, the noise level is measured. The noise level in differential mode is more than the common mode noise. The merit of the two current probes setup is its pre-measurement calibration process for the RF coupling circuit. This method gives good accuracy for the measurement of noise in SMPS.

\section{REFERENCES}

1. Schneider, L.M., 1983. Noise source equivalent circuit model for off-line converters and its use in input filter design. Proc.IEEE.EMC"83 symposium, pp: 167-175.

2. Nave, M.J., 1991. Powerline Filter Design for Switched-Mode Power Supplies. New York: Van No strand Reinhold, pp: 43-48.

3. Jeffrey, P.W., 1993. Electromagnetic Interference, Reduction in Electronics System. PTR Prentice Hall Englewood Cliffs, New Jersey.

4. Mohan,N., 1995. Power Electronics, Converters, Applications and Designs. Second Edn. John Wiley and Sons, New YorK.

5. Zhang, D., D. Chen, M.J. Nave and D. Sable, 1997. Measurement of noise source impedance of off-line converters. Proc.APEC'97 Conf., pp: 918-923.

6. Paul, C.R., 1992. Introduction to Electromagnetic Compatibility. New York: Wiley, pp: 477-486.

7. Ferreira, J.A., P.R. Willcock and S.R. Holm, 1998. Sources, paths and traps of conducted EMI in switch mode circuits. Proc.PESC'98 Conf., 2: 1140-1145.

8. KyeYak, S. and J. Deng, 2004, Measurement of noise source impedance of SMPS using a two probes approach. IEEE Trans. Power Electron., 19: 862-868.

9. www.powerelectronics.com/smps

10. www.powersupply.com/filter http://www.powersupply.com/filter 